\title{
Biomolecular interaction analysis in functional proteomics
}

Daniela Moll, Anke Prinz, Frank Gesellchen, Stephan Drewianka ${ }^{\circ}$, Bastian Zimmermann ${ }^{\circ}$ and Friedrich W. Herberg*

Department of Biochemistry, University of Kassel, Kassel, Germany

and ${ }^{\circ}$ Biaffin $\mathrm{GmbH} \&$ Co KG, Kassel, Germany

* Corresponding author: Friedrich W. Herberg

University of Kassel

Heinrich-Plett-Str. 40, D-34132 Kassel

Phone ++49 (0) 561-804 4511

Fax ++49 (0) 561-804 4466

Email: herberg@uni-kassel.de

Running title: Biomolecular Interaction Analysis 
1 Abbreviations: AlphaScreen, BIA, biomolecular interaction analysis; BRET, bioluminescence resonance energy transfer, cAMP, adenosine- 3', 5'- cyclic monophosphate; C, catalytic subunit of PKA; FP, fluorescence polarization, ITC, isothermal titration calorimetry, PKA, cAMP dependent protein kinase; R, regulatory subunit of PKA; RU, response units; SPR, surface plasmon resonance

KEYWORDS: cAMP dependent protein kinase, surface plasmon resonance, fluorescence polarization, isothermal titration calorimetry, bioluminescence resonance energy transfer, AlphaScreen.

\section{SUMMARY}

To understand the function of highly complex eukaryotic tissues like the human brain, in depth knowledge about cellular protein networks is required. Biomolecular interaction analysis (BIA), as a part of functional proteomics, aims to quantify interaction patterns within a protein network in detail. We used the cAMP dependent protein kinase (PKA) as a model system for the binding analysis between small natural ligands, cAMP and cAMP analogues, with their physiological interaction partner, the regulatory subunit of PKA. BIA comprises a variety of methods based on physics, biochemistry and molecular biology. Here we compared side by side real time SPR (surface plasmon resonance, Biacore), a bead based assay (AlphaScreen), a fluorescence based method (Fluorescence polarisation) and ITC (isothermal titration calorimetry). These in vitro methods were complemented by an in cell reporter assay, $\mathrm{BRET}^{2}$ (bioluminescence resonance energy transfer), allowing to test the effects of cAMP analogues in living cells. 


\section{INTRODUCTION}

The function of biological systems is mediated by proteins and their interactions. Cellular activities are not only dependent on protein expression patterns, but are also controlled by post translational modifications (PTMs), by compartmentalization and protein degradation (Graves and Haystead 2002). In the classical proteomics approach methods like 2D gel electrophoresis and mass spectrometry have been established to describe expression patterns differentially e.g. comparing healthy and diseased tissue. One major goal of functional proteomics is to determine protein-protein, protein-DNA, and protein-ligand interaction with high accuracy and in great detail within a cellular network. Intracellular interaction pathways are influenced to a large extent by PTMs (Shaywitz et al., 2002; Yaqub et al., 2003). The most prominent PTM in an eukaryotic cell is protein phosphorylation, a key event in the regulation of the cell mediated by the action of protein kinases (Manning et al., 2002). About $40 \%$ of all proteins may undergo this crucial PTM during some state of cellular growth and differentiation. The importance of protein phosphorylation in signalling pathways is impressively reflected in the devastating effects of protein kinase dysfunction linked to several human diseases (Blume-Jensen and Hunter 2001; Sachsenmaier 2001) most prominently in human malignancies (Fabbro et al., 2002; Tasken and Aandahl 2004). About 518 Serine/Threonine and Tyrosine specific protein kinases are encoded in the human genome (Manning et al., 2002). Although protein kinases differ substantially in their substrate specificity, activity, biological half life, cellular localisation and function, they share a common overall protein fold. Cyclic AMP dependent protein kinase (PKA) has been used as a model system for kinase function during the last two decades and has been characterized biochemically and structurally in detail (Taylor 1989; Knighton et al., 1991; Taylor et al., 2004). The enzyme consists of a regulatory (R) dimer and two monomeric catalytic (C) subunits forming an inactive holoenzyme complex $\left(\mathrm{R}_{2} \mathrm{C}_{2}\right)$. The holoenzyme complex is activated by increasing concentrations of the second messenger cAMP, a low molecular weight ligand. Upon binding of cAMP to the two distinct binding sites on each R subunit, the $\mathrm{C}$ subunits are released and can phosphorylate substrates in the cytoplasm, but they can also migrate into the nucleus, thus affecting gene regulation via the cAMP response element binding protein (CREB (Hagiwara et al., 1993)) (Figure 1 Cartoon) Not surprisingly, deregulation of PKA activity has been implicated in various human diseases, among them breast cancer, Carney complex and HIV infection (Tasken and Aandahl 2004). PKA has received increasing attention as a factor contributing to neurological disorders. It has already been known that PKA activity is required for memory formation in the mammalian 
hippocampus (Kandel 2001). Recently, it was shown that PKA is also involved in processes concerning the working memory as well as reward-motivated learning, linking PKA action potentially to memory deficits and drug addiction (for review see (Arnsten et al., 2005)).

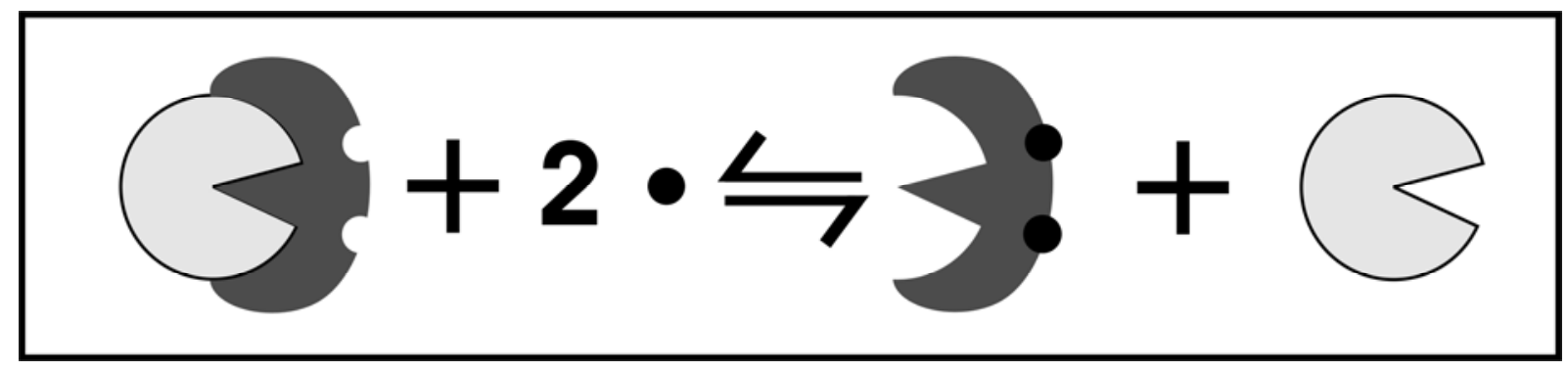

Figure 1 Cartoon Model of PKA holoenzyme activation

The inactive holoenzyme of cAMP dependent protein kinase (PKA) consists of a regulatory subunit dimer ( $\mathrm{R}$ subunit, dark grey) and two catalytic subunits ( $\mathrm{C}$ subunit, light grey). For simplification, only one $\mathrm{C}$ subunit interacting with one $\mathrm{R}$ subunit monomer is shown. Binding of two molecules of cAMP (filled circle) to an R subunit monomer leads to the dissociation of the holoenzyme complex, thereby releasing the active $\mathrm{C}$ subunit.

Due to its modular structure the PKA system is ideally suited as a model for the analyses of protein-protein interaction and protein-small ligand interaction. Here we analyze the binding of the regulatory subunit of PKA to both the natural small ligand CAMP and to the catalytic subunit.

Several methods based on different physico-chemical parameters are available to determine protein-protein interactions. These techniques summarized, under the term biomolecular interaction analysis (BIA), allow a detailed look at molecular interactions. In the following we will describe interactions within the PKA model system determined by surface plasmon resonance (SPR), AlphaScreen, fluorescence polarization (FP), isothermal titration calorimetry (ITC) and bioluminescence resonance energy transfer $\left(\mathrm{BRET}^{2}\right)$. These methods comprise solid phase assays as well as homogenous assays, performed in vitro and in living cells.

\section{MATERIALS AND METHODS}

Reagents. The synthetic peptide substrate Kemptide (LRRASLG) was purchased from Biosyntan GmbH (Berlin, Germany). ATP and NADH were obtained from Biomol GmbH (Hamburg, Germany). 
P11 cation exchanger cellulose and DE52 (Diethylaminoethyl, DEAE) anion exchanger cellulose were obtained from Whatman (Maidstone, UK).

cAMP (adenosine- 3', 5'- cyclic monophosphate),

8AHAcAMP

(8- $(6-$

aminohexyl) aminoadenosine- 3', 5'- cyclic monophosphate),

8CPT-cAMP

(8- (4-

Chlorophenylthio ) adenosine- 3', 5'- cyclic monophosphate),

2Cl-cAMP

Chloroadenosine- 3', 5'- cyclic monophosphate),

6АH-cAMP

(N6- (6-

Aminohexyl)adenosine- 3', 5'- cyclic monophosphate),

8Fluo-cAMP*

(8- [[2-

[(Fluoresceinylthioureido)amino]ethyl]thio]adenosine- 3', 5'- cyclic monophosphate), $\quad 6 \mathrm{Ph}$ cAMP (N6- Phenyladenosine- 3', 5'- cyclic monophosphate), 6MAH-cAMP (N6- ( 6- [N'Methylanthraniloyl]aminohexyl)adenosine- 3', 5'- cyclic monophosphate), 8Br-cAMP (8Bromoadenosine- 3', 5'- cyclic monophosphate), 8Cl-cAMP (8-Chloroadenosine- 3', 5'cyclic monophosphate), 6MB-cAMP (N6- Monobutyryladenosine- 3', 5'cyclic monophosphate), 8NBD-cAMP* (8- [ [2- [ (7- Nitro- 4benzofurazanyl )amino]ethyl]thio]adenosine- 3', 5'- cyclic monophosphate), 8ADOA-cAMP (8- (8- Amino- 3, 6- dioxaoctylamino) adenosine- 3', 5'- cyclic monophosphate), SP8AHAcAMP (8- (6- Aminohexyl) aminoadenosine- 3', 5'- cyclic monophosphorothioate, Spisomer), 8PIP-cAMP (8- Piperidinoadenosine- 3', 5'- cyclic monophosphate), 8AEA-cAMP (8- (2- Aminoethyl) aminoadenosine- 3', 5'- cyclic monophosphate), 2AHA-cAMP (2- (6Aminohexyl) aminoadenosine- 3', 5'- cyclic monophosphate), 2'Mant-cAMP* (2'- O- (NMethylanthraniloyl)adenosine- 3', 5'- cyclic monophosphate), 8Br-cAMP-AM Bromoadenosine- 3', 5'- cyclic monophosphate), Sp8Br-cAMPS (8- Bromoadenosine- 3', 5'cyclic monophosphorothioate), cGMP (Guanosine- 3', 5'- cyclic monophosphate) and cUMP (Uridine- 3', 5'- cyclic monophosphate) were obtained from Biolog Life Science Institute (Bremen, Germany). Fine chemicals (research grade) were purchased from Roth (Karlsruhe, Germany) or from Sigma (Deisenhofen, Germany).

CM5 sensor chips (research grade), NHS (N-hydroxysuccinimide), EDC (N-ethyl-N'(dimethylaminopropyl) -carbodiimide), ethanolamine-HCl, and surfactant P20 were obtained from Biacore AB (Uppsala, Sweden).

\section{Preparation of recombinant proteins.}

The cDNAs for the expression of recombinant proteins were kind gifts from Prof. S. S. Taylor, (murine $\mathrm{C} \alpha$ and bovine $\mathrm{R}_{\alpha}^{\mathrm{I}} \Delta 1-91$ (RI monomer)) University of California, San Diego, USA and Prof. K. Tasken (GST-hR ${ }_{\alpha}^{\mathrm{I}}$ and $\mathrm{hR}_{\alpha}^{\mathrm{II}}$ ), University of Oslo, Oslo, Norway. RI monomer was overexpressed in E. coli BL21 (DE3) and purified according to Herberg et al. using ion exchange chromatography (Herberg et al., 1994). GST-hR ${ }_{\alpha}^{\mathrm{I}}$ was purified using 
Glutathion agarose from Sigma (Deisenhofen, Germany) following standard protocols (Sambrook et al., 2001).

To obtain cAMP free R subunits, the purified protein was incubated with $10 \mathrm{mM}$ cGMP over night at $4^{\circ} \mathrm{C}$. Subsequently, excess cGMP was removed using a PD 10 desalting column (Amersham Biosciences, Freiburg, Germany) followed by extensive dialysis against $150 \mathrm{mM}$ $\mathrm{NaCl}, 20$ mM MOPS, pH 7 (buffer A). To obtain completely nucleotide-free R subunit for ITC measurements, the purified RI monomer was treated with $8 \mathrm{M}$ urea to remove cAMP and then dialyzed as described above (Buechler et al., 1993).

Recombinant PKA C subunit was expressed and purified as described before (Slice and Taylor 1989; Herberg et al., 1993).

The purity of the R subunits was confirmed by SDS-polyacrylamide gel electrophoresis and the biological activity of the proteins was verified using the phosphotransferase assay with the peptide Kemptide (LRRASLG) as a substrate according to Cook et al. (Cook et al., 1982).

AlphaScreen. Biotin labelling of C subunit was performed with a 10:1 molar excess of EZLink NHS Biotin (Perbio Sciences, Bonn, Germany) according to the manufacturer's instructions. The reaction was performed with intact holoenzyme, in order to protect the $\mathrm{C}$ subunit / R subunit interface from chemical modification during the biotinylation procedure. After biotinylation cAMP was added to the reaction mixture to dissociate the holoenzyme complex and the free biotinylated $\mathrm{C}$ subunit was subsequently purified using PKI(5-24) affinity chromatography (Olsen and Uhler 1989).

For the actual AlphaScreen measurements, biotinylated C subunit and GST-tagged R subunit $(0.2 \mathrm{nM}$ each) were mixed together in the presence of serial dilutions of cAMP or $\mathrm{Sp} 8 \mathrm{Br}-$ cAMPS in a 384 well microtiter plate (Optiplate, white, PerkinElmer). In a following step anti-GST acceptor beads and streptavidin donor beads (PerkinElmer, Rodgau, Germany) were added to a final concentration of $20 \mu \mathrm{g} / \mathrm{ml}$. The final reaction volume in each well was $25 \mu \mathrm{l}$ in $25 \mathrm{mM}$ Hepes, pH7.4, $100 \mathrm{mM} \mathrm{NaCl}, 10 \mathrm{mM} \mathrm{MgCl}, 1 \mathrm{mM}$ ATP, $0.1 \%$ BSA. The resulting AlphaScreen signal (counts per second) was determined with a Fusion ${ }^{\mathrm{TM}} \alpha-\mathrm{FP}$ microplate reader (Packard Bioscience, now PerkinElmer) after one hour incubation at room temperature.

Fluorescence Polarization (FP). Two different assay formats were used. In the direct assay format both the RI monomer concentration as well as the 8Fluo-cAMP concentration were varied (10 pM to $1 \mu \mathrm{M}$ for RI monomer and $20 \mathrm{nM}$ to $500 \mathrm{pM}$ for 8Fluo-cAMP). The assay was performed at $20^{\circ} \mathrm{C}$ in buffer A containing $0.005 \%(\mathrm{v} / \mathrm{v})$ CHAPS as a surfactant in a 384 
well microtiterplate (Optiplate, black) using the Fusion ${ }^{\mathrm{TM}} \alpha-\mathrm{FP}$ microplate reader. The fluorescence polarization signal was detected at Ex 485nm / Em FP Filter 535nm with a PMT Voltage of 1100 .

For the fluorescence polarization displacement assay increasing concentrations of cAMP analogues (typically ranging from $1 \mathrm{fM}$ to $1 \mu \mathrm{M}$ ) were mixed with $1 \mathrm{nM}$ 8Fluo-cAMP before adding the RI monomer. Here the concentration of the RI monomer was adapted to $80 \%$ of the maximum value derived from the direct assay using $1 \mathrm{nM}$ 8Fluo-cAMP. Fluorescence polarization was measured after 5 minutes. Data were analyzed with GraphPad Prism 4.0 (GraphPad Software, San Diego, CA) by plotting the resulting polarization signal against the logarithm of the analogue concentration.

Surface plasmon resonance (SPR): All SPR interaction analyses were performed at $20^{\circ} \mathrm{C}$ in buffer A plus $0.005 \%(\mathrm{v} / \mathrm{v})$ surfactant P20 using a Biacore 3000 instrument (Biacore AB, Sweden). For covalent coupling of 8AHA-cAMP, carboxymethylated sensor chip surfaces (CM5, research grade) were activated with NHS / EDC for 10 minutes and 8AHA-cAMP ( $3 \mathrm{mM}$ in $100 \mathrm{mM}$ HEPES, $\mathrm{pH}$ 8.0) was injected for 7 minutes with a flow rate of $5 \mu 1 / \mathrm{min}$. Deactivation of the surface was performed using $1 \mathrm{M}$ ethanolamine- $\mathrm{HCl}(\mathrm{pH}$ 8.5) for 7 minutes. A reference cell was activated accordingly and deactivated subsequently. Competition analyses were performed by injection of $5 \mathrm{nM}$ RI monomer preincubated with varying concentrations of each analogue to be analyzed. The binding signal was monitored for 3 minutes and data points were collected at the end of the association phase. The sensor surfaces were regenerated after each binding cycle by several injections of $3 \mathrm{M}$ guanidinium $\mathrm{HCl}$. After subtracting the reference cell, the resulting binding signals were plotted against the logarithm of each cAMP analogue concentration and an $\mathrm{EC}_{50}$ value was calculated from the dose response curve using GraphPad Prism 3.01 (GraphPad Software, Inc., San Diego/USA).

Isothermal titration calorimetry (ITC). The interaction between RI monomer and cGMP was analyzed in buffer A containing $1 \mathrm{mM} \beta$-mercaptoethanol using a VP-ITC microcalorimeter (MicroCal LLC., Northampton, MA, USA). $5 \mu \mathrm{M}$ RI monomer were allowed to equilibrate in a $1.385 \mathrm{ml}$ cell at $20.0^{\circ} \mathrm{C}$. To ensure that the titrant concentration was at its loading value, two injections of $51.3 \mu \mathrm{M}$ cGMP ( $1 \mu \mathrm{l}$ each) were performed before the actual titration experiment. $5 \mu 1$ injections were conducted until no further binding was observed. Each time the reaction returned to baseline level (approximately after 3 minutes), a new injection was carried out. In order to minimize artifacts, cGMP was dissolved in exactly the same buffer that was used to dialyze the purified protein. For blank subtraction cGMP was injected in identical steps into buffer only. Data evaluation was performed with the software MicroCal 
Origin for ITC (MicroCal LLC., Northampton, MA, USA; see also (Wiseman et al., 1989)), including corrections for volume change during the titration.

BRET $^{2}$ assay: The human $\mathrm{C} \alpha$ coding sequence was amplified using sense and antisense primers harboring unique HindIII and BamHI sites. The fragment was subcloned in-frame into the HindIII/BamHI sites of $\mathrm{pGFP}^{2}-\mathrm{C}$ vector (PerkinElmer). The human $\mathrm{R}_{\alpha}^{\mathrm{II}}$ coding sequence was amplified without the stop codon allowing for cloning with BamHI and KpnI. The fragments were subcloned using the pTrcHis2-Topo ${ }^{\circledR}$ TA cloning kit (Invitrogen, Karlsruhe, Germany Invitrogen; Carlsbad, CA), excised and cloned in-frame into BamHI/KpnI sites of pRluc-N vector (PerkinElmer).

For BRET ${ }^{2}$ assays, COS-7 cells were seeded in a white 96 well microplate (Optiplate, PerkinElmer) at a density of $2 \times 10^{4}$ cells per well. Transfections were carried out in the microplate 24 hours later using $4 \mu$ l Polyfect reagent (Qiagen N.V., Venlo, Netherlands) and a $0.5 \mu \mathrm{g}$ plasmid DNA per well and transfection. 48 hours post transfection, cells were washed with glucose-supplemented Dulbecco's PBS (D-PBS, Invitrogen), and the substrate DeepBlueC ${ }^{\text {TM }}$ (PerkinElmer) was added to a final concentration of $5 \mu \mathrm{M}$ in a total volume of $50 \mu 1$ D-PBS. Light emission was detected using a Fusion ${ }^{\mathrm{TM}} \alpha-\mathrm{FP}$ microplate reader (PerkinElmer; read time 1 second, gain 50). The light output was taken consecutively for each well using filters at $410 \mathrm{~nm}$ wavelength $( \pm 80 \mathrm{~nm}$ bandpass $)$ for the donor and $515 \mathrm{~nm}( \pm 30 \mathrm{~nm}$ bandpass) for the acceptor fluorophore. Background values, routinely obtained with untransfected cells, were subtracted in each measurement. Control transfections with empty pRluc and $\mathrm{pGFP}^{2}$ vectors were carried out with each experiment. BRET ${ }^{2}$ signals were calculated as (emission $515 \mathrm{~nm}^{-}$background $515 \mathrm{~nm}$ ) / (emission $410 \mathrm{~nm}$-background $410 \mathrm{~nm}$ ). GraphPad Prism 4.0 was used for statistical analysis. 


\section{RESULTS}

Biomolecular interaction analysis (BIA) is used to describe binding events between small molecules, proteins (e.g. receptors, enzymes and antibodies), peptides, nucleotides or carbohydrates. Here we focus on one hand on interaction between the PKA regulatory subunit and its LMW ligand cAMP and on the other hand on the influence of cAMP on holoenzyme formation using four in vitro methods and one in cell assay. Equilibrium binding data as well as association and dissociation rate constants were obtained.

\section{AlphaScreen allows compound screening in a homogenous assay format.}

AlphaScreen is a bead-based proximity assay, where a luminescence signal is the readout for a biomolecular interaction. Both interaction partners have to be chemically coupled to latex beads (diameter $250 \mathrm{~nm})$. Upon illumination with laser light $\left(\lambda_{\mathrm{ex}}=680 \mathrm{~nm}\right)$ singlet oxygen $\left(\mathrm{O}_{2}\left({ }^{1} \Delta_{\mathrm{g}}\right)\right)$ is produced on the donor beads (D), see Figure 2 Alpha Screen Inset. If a second interaction partner, coupled to acceptor beads, binds, both beads are brought into close proximity. The chemical energy of the singlet oxygen from the donor beads can diffuse to the acceptor beads, resulting in a luminescence signal $\left(\lambda_{\mathrm{em}}=520-620 \mathrm{~nm}\right)$. If the interaction partners do not bind to each other, the singlet oxygen decays $\left(\mathrm{t}_{1} / 2=4 \mu \mathrm{s}\right)$ and no luminescence signal is observed. The assay is usually performed in a volume of 5-25 $\mu 1$ in 384 well plates and is well suited for automation and thus for high throughput screening.

Immobilization of the interaction partners to donor and acceptor beads, respectively, can be achieved by different strategies. Direct coupling of ligands containing primary amines can be done via reductive amination. However, in the case of PKA R and C subunits this proved to be difficult, potentially due to steric reasons or a loss of activity during the coupling procedure (data not shown). Therefore, an indirect immobilization approache was used, in which biotin labelled $\mathrm{C}$ subunit was captured to streptavidin coated donor beads, while a GST-R subunit fusion protein was immobilized to donor beads via an anti-GST antibody. Special care had to be taken during the biotinylation of the $\mathrm{C}$ subunit, in order to keep the crucial interaction site with the $\mathrm{R}$ subunit interaction free from chemical modifications (see material and methods). In the absence of the activator cAMP this assay setup lead to the generation of a robust luminescence signal reflecting holoenzyme formation by the interaction of $\mathrm{C}$ and $\mathrm{R}$ subunits. The signal was reduced to background levels by increasing cAMP concentrations up to $10 \mu \mathrm{M}$, indicating the complete dissociation of the PKA holoenzyme (see Figure 2 AlphaScreen). The $\mathrm{EC}_{50}$ values derived from the dose-response experiments were slightly higher than those obtained with other methods (see SPR and FP). Screening of cAMP 
analogues demonstrated that their relative potencies were correctly reflected and, consistent within the assay, (Figure 2 AlphaScreen) the $\mathrm{EC}_{50}$ value of Sp8Br-cAMPS was about an order of magnitude higher than the $\mathrm{EC}_{50}$ value of cAMP.

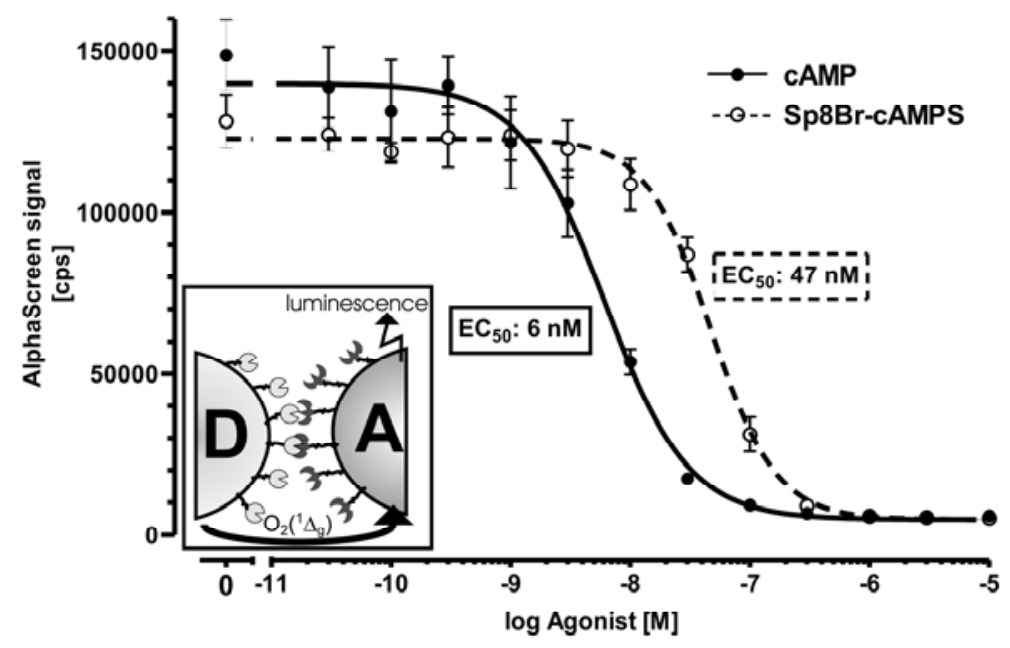

\section{Figure 2 AlphaScreen Detection of PKA holoenzyme dissociation using $\alpha$-Screen}

Biotinylated C subunit and GST-tagged R subunit ( $0.2 \mathrm{nM}$ each) were incubated in a 384 well plate with increasing concentrations of cAMP and Sp8Br-cAMPS, respectively, before adding anti-GST acceptor beads and streptavidin coated donor beads $(20 \mu \mathrm{g} / \mathrm{ml}$ final concentration). Readings were taken after one hour incubation. Data were fitted according to a sigmoidal dose-response model and $\mathrm{EC}_{50}$ values were calculated. All data points represent the mean \pm SD of triplicate measurements. Inset: Depicted in the cartoon is the principle of an AlphaScreen assay. Donor (D) beads were coupled with C subunits (light grey symbol), acceptor (A) beads with R subunits (dark grey symbol). D beads, containing a photosensitizer, release singlet oxygen $\left(\mathrm{O}_{2}\left({ }^{1} \Delta_{\mathrm{g}}\right)\right)$ upon illumination with laser light $\left(\lambda_{\text {ex }} 680 \mathrm{~nm}\right)$. Due to the interaction of $\mathrm{C}$ subunits coupled to the donor beads and $\mathrm{R}$ subunits coupled to the acceptor beads the chemical energy of the singlet oxygen is converted into a luminescence signal $\left(\lambda_{\text {em }}\right.$ 520-620nm). If, upon addition of cAMP, the C and R subunits dissociate, the AlphaScreen signal diminishes.

\section{FP provides a fast and easy setup for high throughput screening}

Fluorescence polarization (FP) is a widely used optical method in a homogenous assay format which allows high throughput screens. It is generally considered to be inexpensive and rapid with a sensitivity close to a classical radioligand binding assay without the need to separate the bound and unbound ligand (Burke et al., 2003; Jameson and Mocz 2005). The theoretical principles underlying polarization measurements have been described previously (Lakowicz 1999; Valeur and Brochon 2001; Jameson et al., 2003; Jameson and Mocz 2005). Briefly, the basic concept of fluorescence polarization is that a fluorophore is preferentially excited when 
it is oriented along the electric vector of the polarized incident light. If the fluorophore does not move, the emitted light is still in the same plane of orientation and therefore remains polarized. Typically, in a watery solution, a fluorophore can rotate freely and therefore the emitted light is no longer polarized. In the experimental setup used here a fluorescence polarization signal can only be detected from fluorophores slowed down drastically in their rotational speed. This occurs for example upon binding of a small fluorescently labelled ligand to a significantly larger molecule. The degree of polarization is determined by measuring the fluorescence intensities of parallel and perpendicular emitted light with respect to the plane of linearly polarized excitation light.

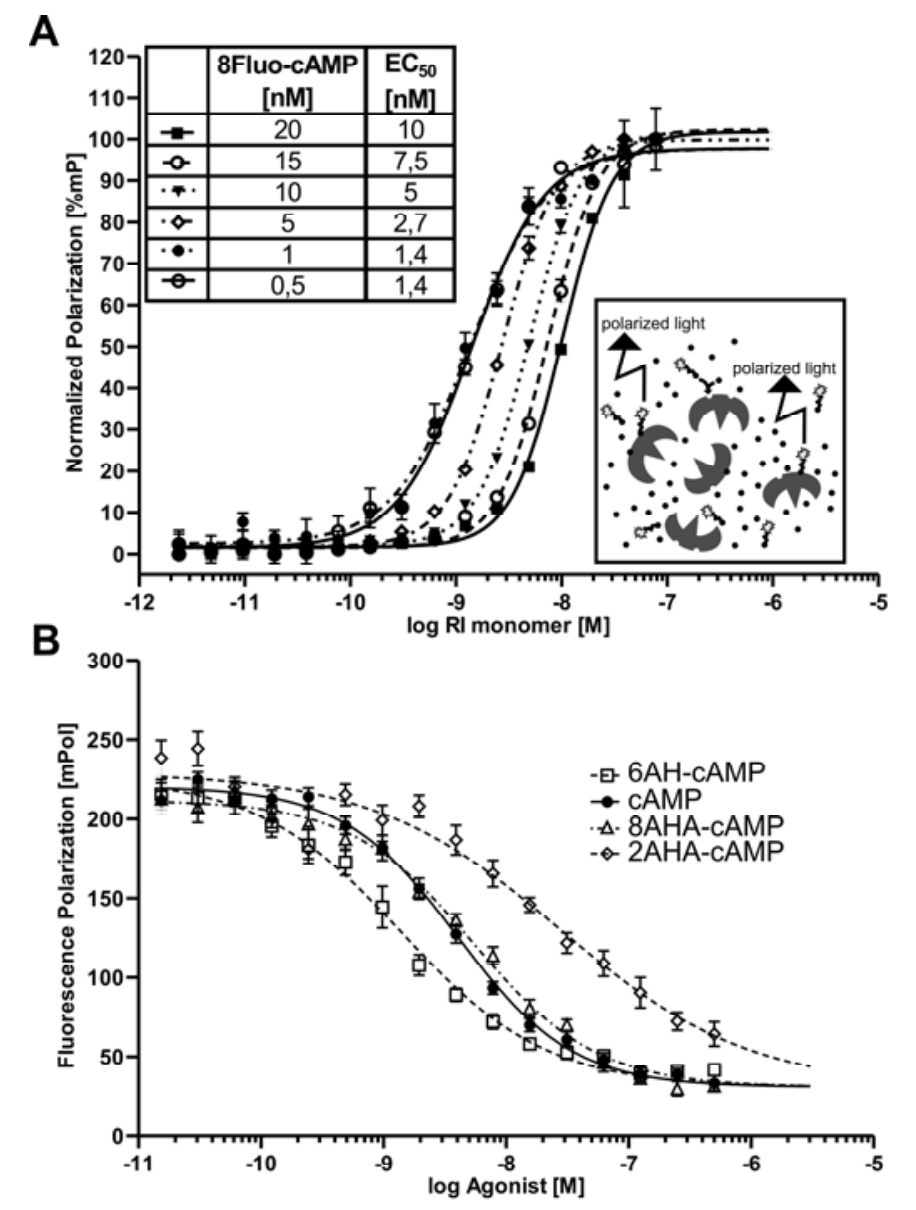

\section{Figure 3 FP Fluorescence polarization}

A. Direct binding of 8Fluo-cAMP to RI monomer. RI monomer was serially diluted in buffer A containing $0,005 \%$ Chaps in a 384 well microtiterplate. 8Fluo-cAMP was added to each well using the concentration indicated on the plot. Each data point represents the mean \pm SEM from triplicate measurements. Inset: Schematic illustration of a FP assay where fluorescently labelled cAMP (dots with stars) and unlabeled cAMP (black dots) compete for binding to the R subunit (dark grey symbol). Only labelled cAMP bound to the R subunit generates a FP signal (black arrow)

B. Displacement experiment. RI monomer $(5 \mathrm{nM})$ was added to a serial dilution of the cAMP analogues indicated on the plot and apparent $\mathrm{EC}_{50}$ were calculated. For experimental details see materials and methods. Each data point represents the mean \pm SEM from six individual wells. 
For the optimization of the assay conditions, the RI monomer concentration was tested over a range of $1 \mathrm{pM}$ to $100 \mathrm{nM}$ with six fixed concentrations of 8Fluo-cAMP.

At higher concentrations $(\geq 5 \mathrm{nM})$ 8Fluo-cAMP is titrated directly to the two RI monomer binding sites. In this case the $\mathrm{EC}_{50}$ values reflect half of the 8Fluo-cAMP concentration consistent with the biological model. At concentrations below $5 \mathrm{nM}$, equilibrium binding measurements can be performed, reflected by the fact, that the apparent $\mathrm{EC}_{50}$ value did not change any further when the ligand concentration was reduced (Figure 3 FP A). The transition between the titration process and equilibrium binding conditions can also be seen in a change of the Hill Slope (Hill slope around 1.8 down to concentrations of $5 \mathrm{nM}$ 8Fluo-cAMP and 1.2 for concentrations below $5 \mathrm{nM}$ ).

For competitive binding experiments (Figure 3 FP B), protein and fluorescent ligand concentrations were held constant. From the direct assay (Figure 3 FP A) the best signal to noise ratio was obtained with $1 \mathrm{nM}$ 8Fluo-cAMP. $5 \mathrm{nM}$ RI monomer, corresponding to $80 \%$ of maximum signal in the direct assay provide a sufficient dynamic range for the competitive assay. Three PKA specific agonists (6AH-cAMP, 8AHA-cAMP and 2AHA-cAMP) and unmodified cAMP were tested. The potency of a given analogue is directly related to the observed $\mathrm{EC}_{50}$ value as shown in Figure $3 \mathrm{FP}$ B. cAMP and 8AHA-cAMP display nearly identical $\mathrm{EC}_{50}$ values ( $4 \mathrm{nM}$ and $5.4 \mathrm{nM}$, respectively), whereas 2AHA-cAMP binds with lower affinity $(27.8 \mathrm{nM}) .6 \mathrm{AH}-\mathrm{AMM}$ has the highest affinity with an $\mathrm{EC}_{50}$ value of $1.4 \mathrm{nM}$.

\section{SPR generates high quality binding data in a wide dynamic range}

Although surface plasmon resonance (SPR) based biosensors are commonly used to directly determine the respective association and dissociation rate constants of a given interaction, this method was expanded here to a solution competition assay format to analyze the relative binding affinities of several cyclic nucleotide derivatives. Cyclic AMP analogues containing an aminohexyl-linker either on the 2-, 6- or 8-position of the adenine ring were covalently immobilised to carboxymethylated dextran surfaces of a CM5 sensor chip at high ligand density. A fixed concentration of $\mathrm{R}$ subunit was injected over the cAMP surfaces in the presence or absence of the respective cAMP derivative as a competitor in solution. The change in SPR signal was monitored in response to various concentrations of a competitor (figure 4 Biacore B). Since the cAMP ligands were immobilised in very high densities, the binding of the $\mathrm{R}$ subunit becomes mainly mass-transfer limited, reflected in a linear increase of the binding signal with time. 

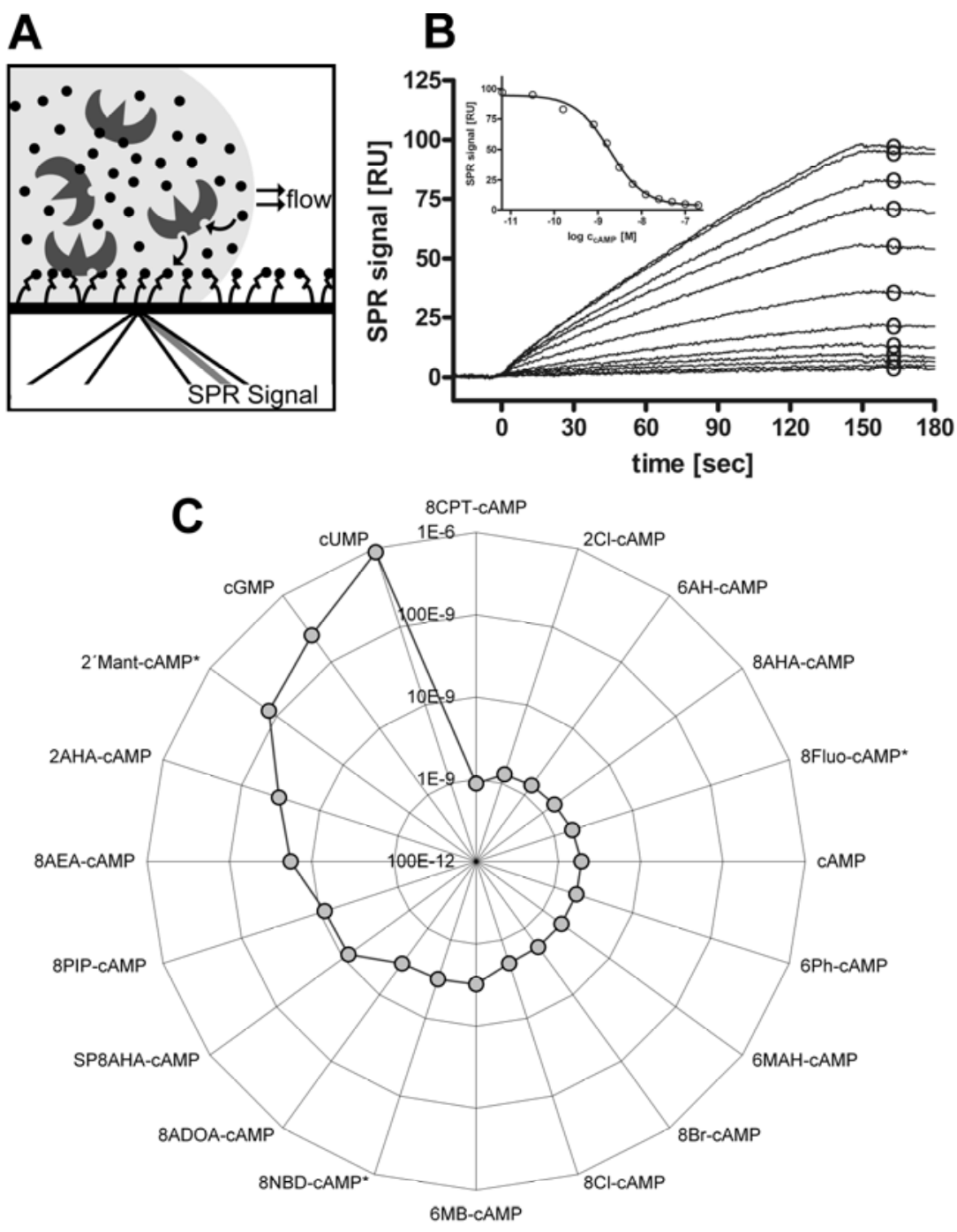

\section{Figure 4 SPR Screening of CAMP analogues in a SPR solution competition assay}

A. Schematic illustration of a solution competition assay performed on a SPR sensor (Biacore setup). cAMP analogues (black dots) compete with immobilized cAMP (black dots linked to surface) for binding to R subunit monomers (dark grey symbol) in the flow phase. Binding is detected by a change in the SPR readout as described elsewhere (Gesellchen et al., 2005).

B. RI monomer $(5 \mathrm{nM})$ was preincubated with cAMP concentrations ranging from $10 \mathrm{pM}$ to $100 \mathrm{nM}$ and the binding to a high density 8AHA-cAMP sensor surface ( $3 \mathrm{mM})$ was followed. The $\mathrm{R}$ subunit was injected for 150 seconds on a Biacore 3000 instrument. 15 seconds after the end of the injection the binding values were recorded (open circle) and the SPR signal was plotted against the logarithm of the cAMP concentration (Inset). The curve was fitted according to a sigmoidal dose response model and an apparent $\mathrm{EC}_{50}$ value was calculated.

C. Apparent $\mathrm{EC}_{50}$ values of different analogues displayed as a radar plot. $\mathrm{EC}_{50}$ values ranging from $10^{-10}-10^{-6} \mathrm{M}$ are plotted in a logarithmic scale as indicated. For abbreviations of the analogues see reagents.

This solution competition approach allows an automated determination of relative affinities for a whole set of cyclic nucleotides binding to different $\mathrm{R}$ subunit isoforms. Here, a set of selected cyclic nucleotides was analyzed for binding to the RI monomer and apparent $\mathrm{EC}_{50}$ values were determined (figure 4 Biacore $\mathrm{B}$ inset) The calculated $\mathrm{EC}_{50}$ values are displayed in 
a radar plot according to their relative affinities (figure 4 Biacore $\mathrm{C}$ ) ranging from subnanomolar values (8CPT-cAMP: $800 \mathrm{pM}$ ) via the nanomolar range (cAMP: $1.7 \mathrm{nM}$ ) to micromolar values (cXMP; $50 \mu \mathrm{M}$, not depicted on the plot).

\section{ITC detects biological interactions without any modification of the interaction partners.}

Using ITC (isothermal titration calorimetry) the heat released (or absorbed) due to ligand binding is directly determined yielding information about the interaction stoichiometry (n), equilibrium binding constant $\left(\mathrm{K}_{\mathrm{A}}\right)$, free energy $(\Delta \mathrm{G})$, enthalpy $(\Delta \mathrm{H})$ and entropy $(\Delta \mathrm{S})$. The experimental setup of an ITC experiment requires some knowledge concerning the nature of the molecules of interest and their inherent binding behaviour.

Wisemann et al. (Wiseman et al., 1989) have shown, that the product of the binding constant $\left(\mathrm{K}_{\mathrm{A}}\right)$, the stoichiometry $(\mathrm{n})$ and the protein concentration has to be in the range between 1 and 1000, preferably between 10 and 100 when performing an direct ITC experiment. Applying the information to the PKA model system, where cAMP binds with high affinity to the R subunit, the optimal protein concentration for the assay has to be rather low (500 pM$250 \mathrm{nM}$ ), which means in turn, that the heat evolved during the reaction at such low protein concentration is well below the detection limit (data not shown). Therefore less potent activator of the PKA holoenzyme, cGMP, was used in the experiment shown in figure 5 ITC Assuming that each RI monomer can bind two molecules cGMP, several different models were applied to fit the binding isotherm. Only a model with a single set of identical sites yielded a satisfying fit (Figure 5 ITC B) and a $\mathrm{K}_{\mathrm{D}}$ value of $117 \mathrm{nM}$ could be calculated. This value was in the same range compared to the data derived from SPR measurements $(250 \mathrm{nM}$, Figure 4 SPR C). Furthermore, based on the Gibbs-Helmholtz equation $(\Delta G=\Delta H-T \Delta S)$, valuable information about the thermodynamics of the interaction can be obtained with ITC. The binding of cGMP to RI monomer was found to be highly exothermic, i.e. with an reaction enthalpy $(\Delta \mathrm{H})$ of $-15.4 \mathrm{kcal} \mathrm{mol}^{-1}$ and an entropy $(\Delta \mathrm{S})$ of $-20 \mathrm{cal} \mathrm{mol}^{-1} \mathrm{~K}^{-1}$. This results in a calculated free enthalpy $(\Delta \mathrm{G})$ of $-9.5 \mathrm{kcal} \mathrm{mol}^{-1}$. 


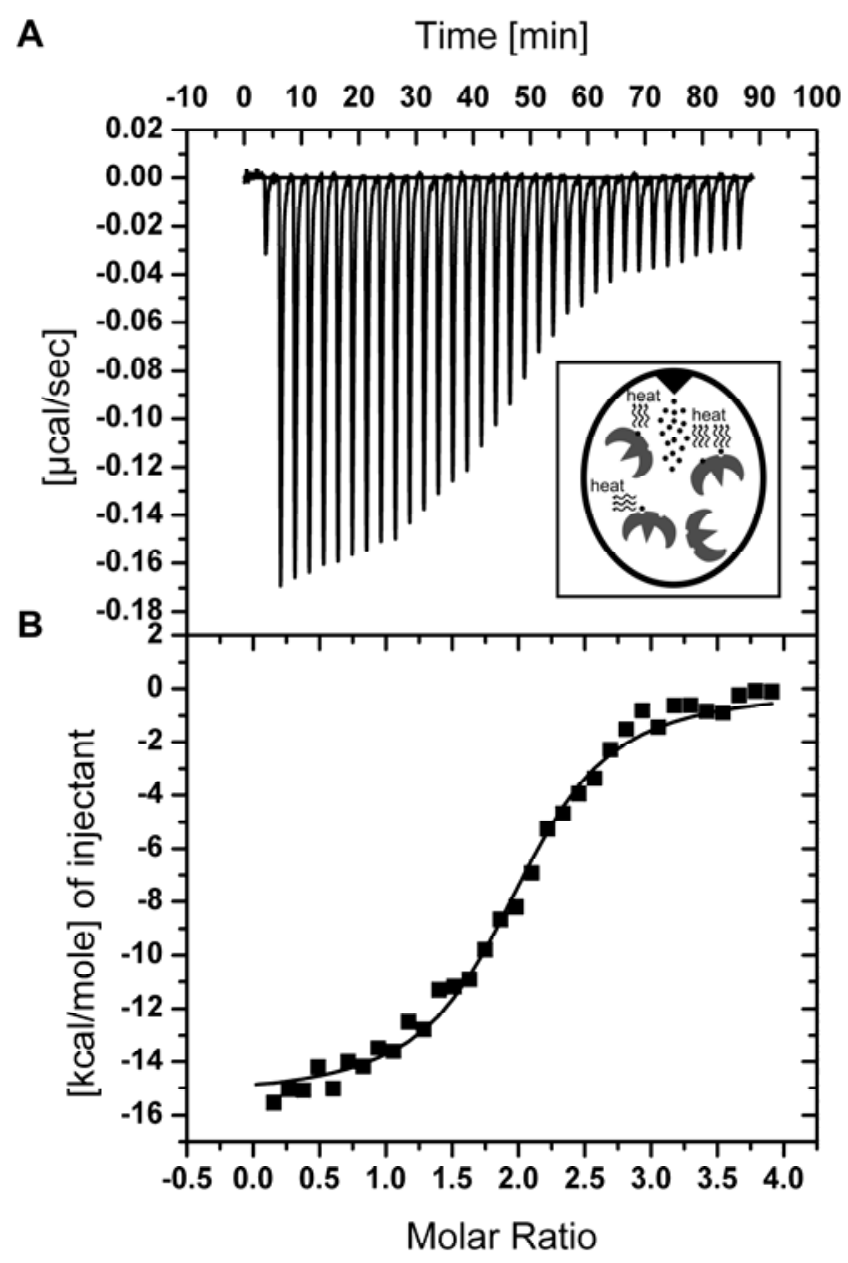

\section{Figure 5 ITC Analysis of cGMP binding to RI monomer employing ITC}

A. Titration of RI monomer $(5 \mu \mathrm{M})$ with $5.1 \mathrm{mM}$ cGMP $(5 \mu 1$ injection steps $)$, raw data. The rate of heat release is plotted as a function of time. Inset: Schematic illustration of an ITC sample cell filled with R subunit (dark grey symbol) and injected cGMP (black dots). As the two interacting partners form a complex, the released heat (arrows) is detected. For simplification the rotating syringe is not pictured.

B. Plot of heat exchange per mole of injectant (integrated areas under the respective peaks in A) vs. molar ratio of protein after blank subtraction (binding isotherm). The best least squares fit applying a model where two cGMP per RI monomer are bound (one binding site model) was performed using the software MicroCal Origin.

\section{cAMP analogues characterization in living cells using BRET ${ }^{2}$}

A genetically encoded sensor was developed to measure PKA subunit dynamics in response to cAMP binding in intact cells. (Prinz et al., manuscript in preparation). The sensor was utilized to characterize the effects of cAMP analogues on the PKA holoenzyme complex in a cellular assay which was adapted to a microplate (96 well) format. Bioluminescence resonance energy transfer occurs when energy generated by substrate oxidation by Rluc is 
transferred to a $\mathrm{GFP}^{2}$ that is in close proximity $(1-10 \mathrm{~nm})$. The BRET ${ }^{2}$ signal is determined by measuring the ratio of green (acceptor, $515 \mathrm{~nm}$ ) over blue (donor, $410 \mathrm{~nm}$ ) light. In the PKA holoenzyme, resonance energy transfer from the luciferase tagged $\mathrm{R}$ subunit to the $\mathrm{GFP}^{2}$ tagged C subunit occurs. However, when cAMP or PKA agonists bind to the holoenzyme, the $\mathrm{BRET}^{2}$ signal decreases in response to increasing ligand concentrations inside the cell. Here, the sensor was used to analyze the efficacy of a membrane permeable PKA agonist, $8 \mathrm{Br}$ cAMP-AM. In detail, COS-7 cells were seeded in a 96 well microplate and co-transfected with plasmid DNAs coding for PKA type II sensor. 48 hours after transfection, cells were washed in D-PBS, and were incubated (15 minutes) with various concentrations of $8 \mathrm{Br}$ cAMP-AM, ranging from 0 to $500 \mu \mathrm{M}$. After addition of the luciferase substrate DeepblueC ${ }^{\mathrm{TM}}$, bioluminescence generated by the luciferase as well as $\mathrm{GFP}^{2}$-fluorescence was quantified using a multi label reader. Figure 6 BRET shows a dose dependent reduction in $\mathrm{BRET}^{2}$ signal in cells expressing PKA type II holoenzyme when treated with up to $500 \mu \mathrm{M}$ analogue (maximum stimulation). From these data, a half maximal effective concentration of about $13 \mu \mathrm{M}$ for 8Br-cAMP-AM was calculated The AM substitution leads to an increase in efficacy by two orders of magnitude in comparison to the parent compound 8Br-cAMP, which has an $\mathrm{EC}_{50}$ value in the millimolar range (about 1.5-2 mM, data not shown).

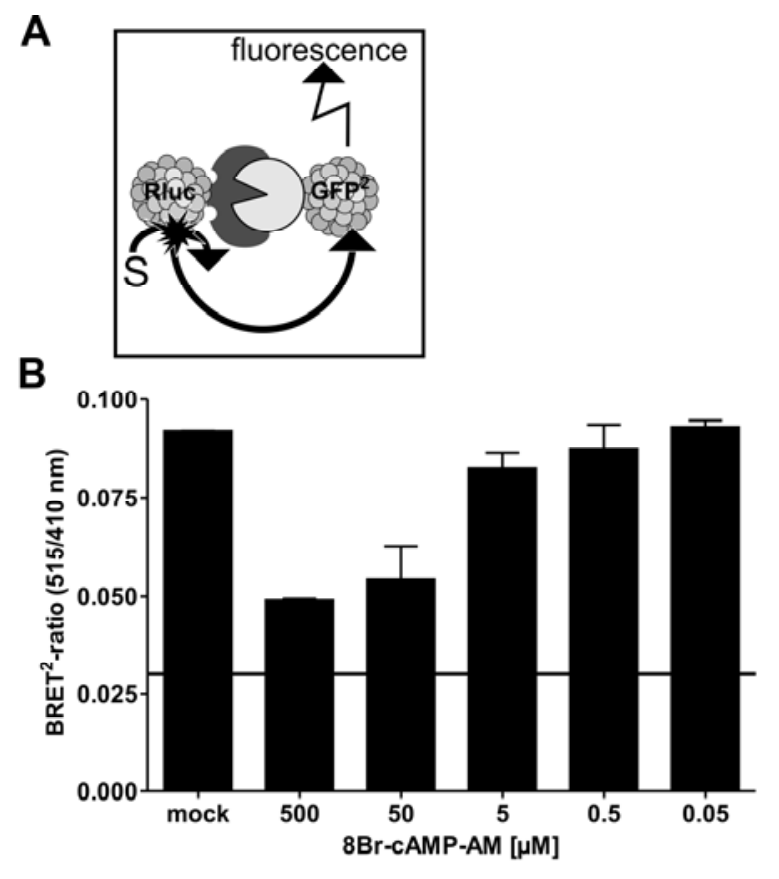

Figure 6 BRET Sensing cAMP in living cells employing BRET².

A. Schematic illustration of bioluminescence resonance energy transfer $\left(\mathrm{BRET}^{2}\right)$. Luminescence is generated upon substrate (S) oxidation by Renilla luciferase (Rluc) and resonance energy is transferred to $\mathrm{GFP}^{2}$ in close proximity $(1-10 \mathrm{~nm})$ causing fluorescence. The interaction of R subunit (dark grey symbol) and C subunit (light grey symbol) can thus be 
monitored, for details see materials and mehods. The BRET ${ }^{2}$-signal decreases when the holoenzyme dissociates in response to rising concentrations of intracellular cAMP or to addition of cAMP analogues to the cells.

B. Cells co-transfected with GFP-C and R ${ }^{\mathrm{II}}$-Rluc were exposed to increasing concentrations of the membrane permeable PKA agonist 8Br-cAMP-AM. After addition of the luciferase substrate DeepBlueC ${ }^{\text {TM}}$, light emission of the donor and the acceptor were determined and $\mathrm{BRET}^{2}$ ratios were calculated. Data are means + S.D. of two independent experimental setups, performed with $n=6$ replicates per analogue at each concentration. A control value (line) was determined with cells co-expressing $\mathrm{GFP}^{2}$ and Rluc proteins solely and represents an average of ten independent experiments. 


\section{DISCUSSION}

The classical proteomics approach comprises the differential description of protein expression patterns using established methods like 2D polyacrylamide gel electrophoresis combined with mass spectrometry. This strategy was expanded by functional proteomics analyzing the distinct interaction patterns of intracellular protein assemblies, and by chemical proteomics trying to construct synthetic entities interfering with previously defined interactions. Recently, interaction networks have been analyzed across an entire genome using for example the tap technology (tandem affinity purification (Gavin et al., 2002)) or a genome wide yeast two hybrid approach (Ho et al., 2002).

Those methods generate a vast amount of binding data and require bioinformatics to interconnect the molecular complexes and pathways within a cellular network, for example the database BIND (Bader et al., 2001). However, these data only provide a qualitative description of a given interaction network. The pharmaceutical industry and biotech research have a strong interest in quantifying the number and strength of intracellular interactions. Furthermore, information about post-translational modifications and subcellular localization have to be included to comprehensively describe cellular networks.

Biological binding events cover a broad range of affinities from low picomolar (e.g. streptavidin-biotin interaction (Green 1990)) via the nanomolar range for ligand receptor- or antibody antigen-binding, to the micromolar range for signalling modules like SH3- or SH2domains. Considering the extremely high local protein concentration mediated by compartmentalization, even affinities in the millimolar range may be of physiological significance. Depending on the situation in the cell, the affinity of a given interaction pair may vary by several orders of magnitude. The $\mathrm{R}$ and $\mathrm{C}$ subunits of PKA for example bind with an affinity of $1 \mathrm{pM}$ in the absence of cAMP which is shifted to $1 \mu \mathrm{M}$ (factor $10^{6}$ !) in the presence of cAMP (Kopperud et al., 2002). Therefore, a high demand for technologies exists capable of analyzing the complete bandwidth of biological affinities. Biomolecular interaction analysis (BIA) combines a variety of assay designs trying to generate highly accurate binding data. In this article established and novel BIA methods are compared side by side using PKA as a model system. AlphaScreen provides a high throughput capable, homogenous, bead based assay platform with luminescence readout. FP, another high throughput method, requires fluorescent labelling of one interaction partner and is used for rapid determination of equilibrium binding constants. SPR using Biacore instruments was chosen as a highly reliable method to monitor association and dissociation kinetics separately. The only truly label free method employed here is ITC generating additional information about the thermodynamics of 
a given interaction. Finally, a cell based assay, BRET ${ }^{2}$, was used to quantify protein-protein interaction in response to low molecular weight (LMW) ligands in living cells. Since every method has certain advantages and inherent drawbacks the following discussion provides a brief assessment of each technology.

\section{AlphaScreen}

AlphaScreen is a robust method for the detection of biological interactions. Because the measurement is time-resolved and light emission occurs at a higher wavelength than excitation, the background is very low resulting in a good signal-to-noise ratio. As opposed to other methods presented here, AlphaScreen is usually not feasible for the determination of affinity constants, since the concentrations of interaction partners on the surfaces of the beads can not be controlled very well. On the other hand, high local concentrations of biomolecules and concurrent avidity effects allow for the detection of low affinity interactions. This results in a high dynamic range from picomolar to millimolar. The method yields reproducible $\mathrm{EC}_{50}$ values and permits an effective screening of compounds that affect binding. This is also reflected in the PKA model system tested here (Figure 2 alpha screen), where the relative potency of a cyclic AMP analogue (Sp8Br-cAMPS) is determined correctly compared to cAMP (Schaap et al., 1993).

In principle, any type of interaction can be measured with AlphaScreen provided that the interaction partners can be immobilized to either donor or to acceptor beads. In contrast to direct coupling of biomolecules to the bead surface one or both interaction partners are commonly captured on antibody-coated beads. Biotinylation of one interaction partner, that can subsequently bind to streptavidin coated beads, is another option. One should be aware of the fact that these indirect methods introduce new equilibria into the interaction, which might influence the measurement.

\section{FP}

Fluorescence polarization (FP) can be used to directly determine $\mathrm{EC}_{50}$ values between fluorescently tagged molecule and its binding partner ideally with a relative molecular weight ratio of 1:50 or higher (Burke et al., 2003). Binding can then be monitored simply by reading the polarization value of the fluorescent molecule after it binds to a larger molecule. Developing competitive binding assays, which can be used to quantify unlabelled competitors in solution, is straightforward (Burke et al., 2003).

The PKA model system can be used after chemical modification of cyclic AMP with a fluorophore. In this study, Fluorescein was attached to the $\mathrm{C} 8$ position of the adenine moiety. Fluorescein is rather big compared to cAMP (cAMP: 329 Da; 8Fluo-cAMP, 816 Da), thus 
adding the fluorophore might significantly affect the interaction with the RI monomer (33.000 Da). Therefore the binding behaviour of fluorescently labelled cAMP was quantified in independent assays. Interestingly, employing the direct FP assay, our results indicate that attaching a fluorophore at the $\mathrm{C} 8$ position does not affect the binding affinity to the RI monomer $\left(\mathrm{EC}_{50} 1.7 \mathrm{nM}\right)$. This was verified by ${ }^{3} \mathrm{H}$-cAMP binding assays (D. Moll, N. C. G. Burghardt, unpublished results, $1.1 \mathrm{nM}$ ) and by SPR experiments (see Figure 4 Biacore C, $1.7 \mathrm{nM}$ ). Furthermore, based on a phosphotransferase activity assay (Cook et al., 1982), the activation constant of the PKA holoenzyme was not altered (D. Moll, unpublished results). Full length $\mathrm{R}^{\mathrm{I}}$ and $\mathrm{R}^{\mathrm{II}}$ subunits of PKA purified from bovine skeletal muscle and heart, respectively, confirmed that 8Fluo-cAMP and cAMP bind comparably well (MucignatCaretta and Caretta 1997).

$\mathrm{EC}_{50}$ values for the analogues 2AHA-cAMP and 6AH-cAMP are in perfect agreement with the SPR competition results (2AHA-cAMP: SPR $33 \mathrm{nM}$, FP $27.8 \mathrm{nM}$; 6AH-cAMP: $1.4 \mathrm{nM}$ for both SPR and FP (Figure 4 Biacore C \& Figure 3 FP B)). 8AHA-cAMP and cAMP show similar affinities in both assays with slightly higher $\mathrm{EC}_{50}$ values generated with FP (cAMP: SPR 1.7 nM, FP 4 nM; 8AHA-cAMP: SPR 1.5 nM, FP 5.4 nM; Figure 4 Biacore C \& Figure 3 FP B).

FP assays have been developed for nearly all major classes of drug targets, including GPCR's (G-Protein coupled receptors), kinases, phosphatases, proteases and nuclear receptors. (Burke et al., 2003).

\section{SPR}

SPR measurements have been utilized in the drug development process and can also be used in functional proteomics in order to kinetically characterize binding events within cellular interaction networks. Typical applications are target characterization and validation of preselected binder in secondary screens. Furthermore, lead optimization, QSAR (quantitative structure activity relationship) and early ADME studies (adsorption, distribution, metabolism, excretion) can be performed. Employing SPR almost every biomolecular interaction can be measured. In a Biacore system not only equilibrium binding data but also rate constants for the association and dissociation can be determined allowing the characterization of a wide range of affinities with high reproducibility, high sensitivity and low sample consumption.

Potential limitations of SPR biosensors lie in the detection principle: a mass change close to the sensor surface is converted into an optical signal, meaning that a small mass increase results in an accordingly small signal. Consequently the immobilization of the low molecular weight interaction partner appears to be favourable. Immobilization of small ligands, 
however, often requires their derivatization which may affect the functionality of a given ligand.

A general problem of solid phase assays is a reduction in degrees of freedom upon immobilization, which can severely influence or even prevent binding due to steric hindrance.

Although SPR based biosensors are commonly used in the direct assay format, alternatively the binding of low molecular weight substances can be assessed in solution or surface competition assays as shown here also for AlphaScreen and FP. In the solution competition setup, the competitor molecule interferes with the binding of the analyte to the immobilized ligand, where in the surface competition experiment the molecule of interest competes with the analyte for the same binding site. In work presented here, the relative binding affinities ( $\mathrm{EC}_{50}$ values Figure 4 Biacore $\mathrm{C}$ ) of several cyclic nucleotide derivatives containing different functional modifications were tested in form of a solution competition assay. This also avoided problems arising from steric hindrance and from mass transfer limitations (Glaser 1993). With this approach it was possible to cover affinities of several orders of magnitude. Still, depending on the position where the cyclic nucleotide was immobilized to the sensor surface, steric effects had to be considered. However, control experiments demonstrated that immobilizing cyclic AMP via aminohexyl linkers at the two, six or eight position of the adenine ring did not influence the $\mathrm{EC}_{50}$ values (data not shown).

\section{ITC}

Isothermal titration calorimetry (ITC) has been applied to a variety of biochemical interactions. In contrast to all techniques discussed so far no chemical modification of the target molecule is needed for immobilization or labelling. Besides equilibrium binding data ITC delivers thermodynamic parameters such as enthalpy $(\Delta \mathrm{H})$, entropy $(\Delta \mathrm{S})$ and the stoichiometry (n). However ITC may require rather large amounts of the interaction partners especially of the molecule in the syringe, which is usually used in $7 \bullet n-$ fold molar excess. The interaction between RI monomer and the natural effector cGMP was shown to be highly exothermic. This allowed to measure cGMP / R subunit interaction at relatively low protein concentration using less than $6 \mu \mathrm{M}$ RI monomer.

(Gorshkova et al., 1995; Lin and Lee 2002) could demonstrate that cGMP binding to the two nucleotide binding sites in the E. coli catabolite activator protein (CAP) is also exothermic $\left(\Delta \mathrm{H}=-1.7,-2,7 \mathrm{kcal} \mathrm{mol}^{-1}\right)$, however, 10-fold less compare to RI monomer $(\Delta \mathrm{H}=$ $\left.15.4 \mathrm{kcal} \mathrm{mol}^{-1}\right)$. Accordingly a much higher concentration of CAP protein $(50-400 \mu \mathrm{M})$ was required for ITC experiments. Interestingly the cGMP binding to CAP is entropically 
favoured with a positive around $\Delta \mathrm{S}=+13 \mathrm{kcal} \mathrm{mol}^{-1} \mathrm{~K}^{-1}$ (Gorshkova et al., 1995; Lin and Lee 2002) in contrast to cGMP binding to RI monomer with an unfavourable negative $\Delta \mathrm{S}=$ $20.8 \mathrm{kcal} \mathrm{mol}^{-1} \mathrm{~K}^{-1}$.

\section{BRET $^{2}$}

With the use of GFP as well as luciferases from several organisms as genetically encoded sensors in living cells, two main resonance energy based methods (FRET and BRET) for the investigation of protein-protein interactions have emerged. For a detailed comparison refer to (Boute et al., 2002; Xu et al., 2003). In contrast to the traditional yeast two hybrid assay, where protein-protein interactions are commonly performed in the nucleus, FRET and BRET based biomolecular interactions can be monitored in defined sub-cellular compartments. In our study we chose a BRET ${ }^{2}$ based reporter system to quantify holoenzyme dissociation in vivo, because $\mathrm{BRET}^{2}$ represents a ratiometric assay. $\mathrm{BRET}^{2}$ can be used with a defined population of cells as well as in cell lysates (not shown). Compounds influencing holoenzyme formation were investigated and tested for efficacy and bioavailability, complementing established in vitro assays. With this BRET $^{2}$ reporter system, we here investigated the effect of the membrane permeable agonist 8Br-cAMP-AM on intracellular PKA type II holoenzyme, which results in a dose dependent $\mathrm{BRET}^{2}$ signal reduction following the dissociation of the holoenzyme. 8Br-cAMP-AM is far more potent (about 50 times) compared to its parent compound $8 \mathrm{Br}-\mathrm{cAMP}$ (data not shown), with an $\mathrm{EC}_{50}$ value of $13 \mu \mathrm{M}$ (Figure 6 BRET). The data are in good agreement with earlier results testing the potency of acetoxymethyl esters of $\mathrm{Bt}_{2}$-cAMP using physiological tests and microinjected PKA type II FRET sensors (Schultz et al., 1993). $\mathrm{EC}_{50}$ data in the same range were reported in antiproliferative studies with several acetoxymethylesters of cAMP (Bartsch et al., 2003) and in studies on the anti-apoptotic properties of 8Br-cAMP-AM in neutrophils challenged with TNF- $\alpha$ (Krakstad et al., 2004).

BRET based assays can be easily upgraded to medium high throughput format under standardized conditions. With the development of novel microscopic and imaging techniques, resonance energy based methods could be useful for mapping signalling events in cells and even living organisms (Tsien 2003).

\section{ACKNOWLEDGEMENTS}

We thank Angelika Wattrodt, Mandy Diskar and Michael Krieg (University of Kassel, Germany) for superb technical help and Prof. Susan Taylor (University of San Diego, USA) and Dr. Frank Schwede, Dr. Gottfried Genieser (Biolog, Bremen) for providing vector DNA 
and cyclic nucleotide analogues, respectively. We acknowledge Dr. Claudia Hahnefeld and Dr. Christian Hammann (University of Kassel, Germany) for helpful discussions regarding SPR and ITC measurements. This work was supported by grants of the Deutsche Forschungsgemeinschaft (DFG, He1818/4) and the European Commission (EU-RTD QLK3CT-2002-02149) and by the German Ministry of Education and Research (BMBF PPOS22T02) to F. W. Herberg.

\section{REFERENCES}

Arnsten AF, Ramos BP, Birnbaum SG, Taylor JR (2005) Protein kinase A as a therapeutic target for memory disorders: rationale and challenges. Trends Mol Med 11(3): 121128.

Bader GD, Donaldson I, Wolting C, Ouellette BF, Pawson T, Hogue CW (2001) BIND-The Biomolecular Interaction Network Database. Nucleic Acids Res 29(1): 242-245.

Bartsch M, Zorn-Kruppa M, Kuhl N, Genieser HG, Schwede F, Jastorff B (2003) Bioactivatable, membrane-permeant analogs of cyclic nucleotides as biological tools for growth control of C6 glioma cells. Biol Chem 384(9): 1321-1326.

Blume-Jensen P, Hunter T (2001) Oncogenic kinase signalling. Nature 411(6835): 355-365.

Boute N, Jockers R, Issad T (2002) The use of resonance energy transfer in high-throughput screening: BRET versus FRET. Trends Pharmacol Sci 23(8): 351-354.

Buechler JA, Taylor SS (1990) Differential labeling of the catalytic subunit of cAMPdependent protein kinase with a water-soluble carbodiimide: identification of carboxyl groups protected by MgATP and inhibitor peptides. Biochemistry 29(7): 1937-1943.

Buechler YJ, Herberg FW, Taylor SS (1993) Regulation-defective mutants of type I cAMPdependent protein kinase. Consequences of replacing arginine 94 and arginine 95. J Biol Chem 268(22): 16495-16503.

Burke TJ, Loniello KR, Beebe JA, Ervin KM (2003) Development and application of fluorescence polarization assays in drug discovery. Comb Chem High Throughput Screen 6(3): 183-194.

Cook PF, Neville ME, Jr., Vrana KE, Hartl FT, Roskoski R, Jr. (1982) Adenosine cyclic 3',5'monophosphate dependent protein kinase: kinetic mechanism for the bovine skeletal muscle catalytic subunit. Biochemistry 21(23): 5794-5799.

Fabbro D, Ruetz S, Buchdunger E, Cowan-Jacob SW, Fendrich G, Liebetanz J, Mestan J, O'Reilly T, Traxler P, Chaudhuri B, Fretz H, Zimmermann J, Meyer T, Caravatti G, Furet P, Manley PW (2002) Protein kinases as targets for anticancer agents: from inhibitors to useful drugs. Pharmacol Ther 93(2-3): 79-98.

Gavin AC, Bosche M, Krause R, Grandi P, Marzioch M, Bauer A, Schultz J, Rick JM, Michon AM, Cruciat CM, Remor M, Hofert C, Schelder M, Brajenovic M, Ruffner H, Merino A, Klein K, Hudak M, Dickson D, Rudi T, Gnau V, Bauch A, Bastuck S, Huhse B, Leutwein C, Heurtier MA, Copley RR, Edelmann A, Querfurth E, Rybin V, Drewes G, Raida M, Bouwmeester T, Bork P, Seraphin B, Kuster B, Neubauer G, Superti-Furga $G$ (2002) Functional organization of the yeast proteome by systematic analysis of protein complexes. Nature 415(6868): 141-147.

Gesellchen F, Zimmermann B, Herberg FW (2005) Direct optical detection of protein-ligand interactions. Methods Mol Biol 305: 17-46.

Glaser RW (1993) Antigen-antibody binding and mass transport by convection and diffusion to a surface: a two-dimensional computer model of binding and dissociation kinetics. Anal Biochem 213(1): 152-161. 
Gorshkova I, Moore JL, McKenney KH, Schwarz FP (1995) Thermodynamics of cyclic nucleotide binding to the cAMP receptor protein and its T127L mutant. J Biol Chem 270(37): 21679-21683.

Graves PR, Haystead TA (2002) Molecular biologist's guide to proteomics. Microbiol Mol Biol Rev 66(1): 39-63; table of contents.

Green NM (1990) Avidin and streptavidin. Methods Enzymol 184: 51-67.

Hagiwara M, Brindle P, Harootunian A, Armstrong R, Rivier J, Vale W, Tsien R, Montminy MR (1993) Coupling of hormonal stimulation and transcription via the cyclic AMPresponsive factor CREB is rate limited by nuclear entry of protein kinase A. Mol Cell Biol 13(8): 4852-4859.

Herberg FW, Bell SM, Taylor SS (1993) Expression of the catalytic subunit of cAMPdependent protein kinase in Escherichia coli: multiple isozymes reflect different phosphorylation states. Protein Eng 6(7): 771-777.

Herberg FW, Dostmann WR, Zorn M, Davis SJ, Taylor SS (1994) Crosstalk between domains in the regulatory subunit of cAMP-dependent protein kinase: influence of amino terminus on cAMP binding and holoenzyme formation. Biochemistry 33(23): 7485-7494.

Ho Y, Gruhler A, Heilbut A, Bader GD, Moore L, Adams SL, Millar A, Taylor P, Bennett K, Boutilier K, Yang L, Wolting C, Donaldson I, Schandorff S, Shewnarane J, Vo M, Taggart J, Goudreault M, Muskat B, Alfarano C, Dewar D, Lin Z, Michalickova K, Willems AR, Sassi H, Nielsen PA, Rasmussen KJ, Andersen JR, Johansen LE, Hansen LH, Jespersen H, Podtelejnikov A, Nielsen E, Crawford J, Poulsen V, Sorensen BD, Matthiesen J, Hendrickson RC, Gleeson F, Pawson T, Moran MF, Durocher D, Mann M, Hogue CW, Figeys D, Tyers M (2002) Systematic identification of protein complexes in Saccharomyces cerevisiae by mass spectrometry. Nature 415(6868): 180-183.

Jameson DM, Croney JC, Moens PD (2003) Fluorescence: basic concepts, practical aspects, and some anecdotes. Methods Enzymol 360: 1-43.

Jameson DM, Mocz G (2005) Fluorescence polarization/anisotropy approaches to study protein-ligand interactions: effects of errors and uncertainties. Methods Mol Biol 305: 301-322.

Kandel ER (2001) The molecular biology of memory storage: a dialogue between genes and synapses. Science 294(5544): 1030-1038.

Knighton DR, Zheng JH, Ten Eyck LF, Xuong NH, Taylor SS, Sowadski JM (1991) Structure of a peptide inhibitor bound to the catalytic subunit of cyclic adenosine monophosphate-dependent protein kinase. Science 253(5018): 414-420.

Kopperud R, Christensen AE, Kjarland E, Viste K, Kleivdal H, Doskeland SO (2002) Formation of inactive cAMP-saturated holoenzyme of cAMP-dependent protein kinase under physiological conditions. J Biol Chem 277(16): 13443-13348.

Krakstad C, Christensen AE, Doskeland SO (2004) cAMP protects neutrophils against TNFalpha-induced apoptosis by activation of cAMP-dependent protein kinase, independently of exchange protein directly activated by cAMP (Epac). J Leukoc Biol 76(3): 641-647.

Lakowicz JR (1999). Fluorescence Anisotropy. Principles of fluorescence Spectroscopy. New York, Kluwer academic/Plenum Publishers: 291-319.

Lin SH, Lee JC (2002) Communications between the high-affinity cyclic nucleotide binding sites in E. coli cyclic AMP receptor protein: effect of single site mutations. Biochemistry 41(39): 11857-11867.

Manning G, Whyte DB, Martinez R, Hunter T, Sudarsanam S (2002) The protein kinase complement of the human genome. Science 298(5600): 1912-1934. 
Mucignat-Caretta C, Caretta A (1997) Binding of two fluorescent cAMP analogues to type I and II regulatory subunits of cAMP-dependent protein kinases. Biochim Biophys Acta 1357(1): 81-90.

Olsen SR, Uhler MD (1989) Affinity purification of the C alpha and C beta isoforms of the catalytic subunit of cAMP-dependent protein kinase. J Biol Chem 264(31): 1866218666.

Sachsenmaier C (2001) Targeting protein kinases for tumor therapy. Onkologie 24(4): 346355.

Sambrook J, Russel DW, Irwin N, Janssen KA (2001) Molecular Cloning - A laboratory manual. 3rd ed., Cold Spring Harbor Laboratory Press, New York.

Schaap P, van Ments-Cohen M, Soede RD, Brandt R, Firtel RA, Dostmann W, Genieser HG, Jastorff B, van Haastert PJ (1993) Cell-permeable non-hydrolyzable cAMP derivatives as tools for analysis of signaling pathways controlling gene regulation in Dictyostelium. J Biol Chem 268(9): 6323-6331.

Schultz C, Vajanaphanich M, Harootunian AT, Sammak PJ, Barrett KE, Tsien RY (1993) Acetoxymethyl esters of phosphates, enhancement of the permeability and potency of cAMP. J Biol Chem 268(9): 6316-6322.

Shaywitz AJ, Dove SL, Greenberg ME, Hochschild A (2002) Analysis of phosphorylationdependent protein-protein interactions using a bacterial two-hybrid system. Sci STKE 2002(142): PL11.

Slice LW, Taylor SS (1989) Expression of the catalytic subunit of cAMP-dependent protein kinase in Escherichia coli. J Biol Chem 264(35): 20940-20946.

Tasken K, Aandahl EM (2004) Localized effects of cAMP mediated by distinct routes of protein kinase A. Physiol Rev 84(1): 137-167.

Taylor SS (1989) cAMP-dependent protein kinase. Model for an enzyme family. J Biol Chem 264(15): 8443-8446.

Taylor SS, Yang J, Wu J, Haste NM, Radzio-Andzelm E, Anand G (2004) PKA: a portrait of protein kinase dynamics. Biochim Biophys Acta 1697(1-2): 259-269.

Tsien RY (2003) Imagining imaging's future. Nat Rev Mol Cell Biol Suppl: SS16-21.

Valeur B, Brochon JC, Eds. (2001). New Trends in Fluorescence Spectroscopy - Applications to Chemical and Life Sciences. Springer Series on Fluorescence - Methods and Applications. Heidelberg, Springer Verlag.

Wiseman T, Williston S, Brandts JF, Lin LN (1989) Rapid measurement of binding constants and heats of binding using a new titration calorimeter. Anal Biochem 179(1): 131-137.

Xu Y, Kanauchi A, von Arnim AG, Piston DW, Johnson CH (2003) Bioluminescence resonance energy transfer: monitoring protein-protein interactions in living cells. Methods Enzymol 360: 289-301.

Yaqub S, Abrahamsen H, Zimmermann B, Kholod N, Torgersen KM, Mustelin T, Herberg FW, Tasken K, Vang T (2003) Activation of C-terminal Src kinase (Csk) by phosphorylation at serine-364 depends on the Csk-Src homology 3 domain. Biochem J 372(Pt 1): 271-278. 